\title{
The HI in the Large Barred Spiral NGC 1365
}

\author{
S. Jörsäter
}

Stockholm Observatory S-13336 Saltsjöbaden, Sweden

G. A. van Moorsel

Array Operations Center, National Radio Astronomy Observatory Socorro, New Mexico, 87801, USA

\begin{abstract}
.
We have made high resolution HI observations using the $\mathrm{VLA}^{1}$ of the barred spiral galaxy NGC 1365 . This galaxy contains $15.2 \times 10^{9} M_{\odot}$ of HI. The velocity field is strongly affected by the bar only in the inner parts. NGC 1365 has a warp and it has 4 well developed spiral arms and 2 rudimentary ones. NGC 1365 has an unusually dropping rotation curve.
\end{abstract}

\section{Introduction}

NGC 1365 is an ideal galaxy for the study of kinematics and dynamics in barred spirals - it has no companions, it is symmetric and has a strong bar, it has an inclination small enough to allow good overview of the structure and at the same time large enough to guarantee an appreciable line of sight component of the rotation velocities (Jörsäter 1984). This paper constitutes a brief summary of a more complete presentation (Jörsäter \& Van Moorsel 1995). We have mapped NGC 1365 in the HI using the VLA at sufficiently high resolution to allow us to study the detailed gas flow in the arms and in the bar. The channel separation was $20.6 \mathrm{~km} \mathrm{~s}^{-1}$. We used the VLA in the $\mathrm{BnA}, \mathrm{CnB}$ and $\mathrm{DnC}$ configurations.

\section{HI Morphology}

The total HI map at a resolution of $11^{\prime \prime} 5 \times 66^{\prime \prime} 3$ and a Johnson $B$ image made from a mosaic of CCD images obtained with the Danish $1.5 \mathrm{~m}$ telescope at ESO, La Silla using a focal reducer and a TI $800 \times 800$ CCD are shown in Figure 1 at the same scale. NGC 1365 contains $15.2 \times 10^{9} M_{\odot}$ of HI. From the HI map we note the following :

1) There is no HI component that delineates the bar. The only exception to this is the small armlet to the northeast and very close to the nucleus. This

\footnotetext{
${ }^{1}$ The VLA is operated by Associated Universities, Inc., under contract with the National Science Foundation
} 

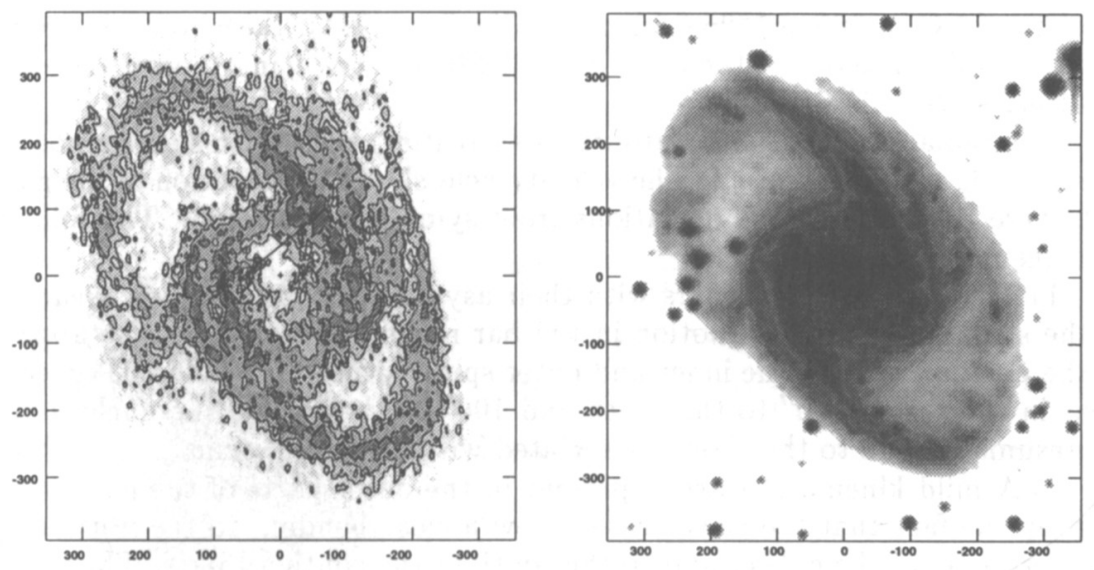

Figure 1. The total hydrogen map of NGC 1365 (left) and a Johnson $B$ image (right). HI contours are at $25,50,100$ and $200 \mathrm{Jy} \mathrm{m} \mathrm{s}^{-1} /$ beam. The lowest contour corresponds to $4.1 \times 10^{-20}$ atoms $\mathrm{cm}^{-2}$ or $3.3 M_{\odot}$ $\mathrm{pc}^{-2}$. Both axes are in units arcseconds from the nucleus.

feature coincides with the dust lane in the bar. But the bar itself does not seem to contain any appreciable amounts of HI. The CO observations of NGC 1365 by Sandqvist et al. (1988) and Sandquist et al. (1994) clearly show that the gas in the bar region is indeed mainly in molecular form. They derive a molecular gas mass of $20 \times 10^{9} M_{\odot}$ in the nuclear and central bar region of NGC $1365-$ a mass similar to that we find for the total HI.

2) NGC 1365 is a multi-armed galaxy and the morphology of the HI in general in the spiral arms follows closely that of the optical emission. The brighter HII complexes frequently have HI counterparts. On the west side we clearly see $3 \mathrm{arms}$ in the HI emission (and they are also hinted at in the optical; in unsharp masked images they appear quite clearly). NGC 1365 is thus a (five to) six armed galaxy.

3) There are two noteworthy exceptions from the mentioned agreement between the HI and the optical morphology. The first is the extension of the HI to the north. The other is the appearance of a sharp cutoff in the HI we see at the west and north-west side. This cutoff follows almost a straight edge. The optical emission shows no sign of a similar cutoff.

4) The HI surface density shows a rather sharp drop in the outer areas except in the northern extension. The HI ends just outside the Holmberg radius (about $340^{\prime \prime}$ or $34 \mathrm{kpc}$ ) except in the northern extension. This indicates that NGC 1365 has a compact HI disk.

\section{HI kinematics}

We find a systemic heliocentric velocity of $1632 \pm 3 \mathrm{~km} \mathrm{~s}^{-1}$ for the kinematic symmetry center (nucleus) which is in good agreement with the optical radial 
velocity of NGC 1365 of $1626 \pm 5 \mathrm{~km} \mathrm{~s}^{-1}$ (Jörsäter 1984). Based on this, we have adopted a distance of $20 \mathrm{Mpc}$ to NGC 1365 (Jörsäter \& Van Moorsel 1995); The resulting scale is $1^{\prime \prime} \sim 100 \mathrm{pc}$.

The radial velocity field contours (isovelocities) are shown superimposed on the total HI map in Figure 2. The velocity field shows the rotation of the galaxy but there are also obvious deviations from symmetric rotation. The following features may be noted :

1) The isovelocity contours with their asymmetric wiggles show clear signs of the strong non-circular motion in the bar region. Wiggles are also apparent at the position of both the inner and outer spiral arms - the effect is especially clear in the region $100^{\prime \prime}$ to the south and $100^{\prime \prime}$ to the east of the nucleus. This is presumably due to the shocks associated with the spiral arms.

2) A mild kinematic warp is present in the outer parts of the galaxy. It is seen by the fact that the isovelocities show a clear bending to the north in the northern part of the galaxy and to the south in the southern part. This warp is situated well within the spiral arm region.

\section{Discussion}

It is difficult to distinguish the influence of indications of non-flatness or disturbed velocities such as warps on the one hand, and of the non-circular motions caused by the bar on the other hand. Rather strong non-circular motions are seen to be present in the bar region, as expected, but this is mainly within $70^{\prime \prime}$ from the nucleus. The warp perturbations, on the other hand, start to be noticeable at a distance of about $220^{\prime \prime}$ from the nucleus. It is not very likely that the bar should have a substantial influence on the velocity field at these large radii.

We have built tilted ring models of the velocity field of NGC 1365 , with which we successfully have modeled the warp. It starts at a rather small relative radius, at $250^{\prime \prime}$ from the nucleus which corresponds to $70 \%$ of the $R_{25}$. In an analysis following Briggs (1990) we find that the twist angle changes from $270^{\circ}$ to almost $330^{\circ}$ but only the second half of this range is significant since the warp angle (inclination relative to the central disk) is very small in the inner parts. The warp angle goes from $0^{\circ}$ to $16^{\circ}$ and then decreases back to about $11^{\circ}$ after which it starts increasing again.

The rotation curve of NGC 1365, shown in Figure 2, is dropping substantially, from about $300 \mathrm{~km} \mathrm{~s}^{-1}$ to less than $200 \mathrm{~km} \mathrm{~s}^{-1}$ or to $63 \%$ of the peak velocity. The sharply dropping rotation curve of NGC 1365 makes it, to our knowledge, unique. It begins to fall far inside the $\mathbf{R}_{25}$ radius. The falling trend continues as far as it can be traced. Figure 2 also compares this rotation curve to a corresponding Keplerian curve. It is quite clear that the rotation curve of NGC 1365 is well represented by a Keplerian curve from $240^{\prime \prime}$ to the measured limit at $400^{\prime \prime}$, i.e. over $40 \%$ of its radius! The corresponding mass as derived from this simple model is $3.9 \times 10^{11} M_{\odot}$ resulting in a very reasonable $\mathrm{M} / \mathrm{L}_{B}$ of 4.8. We may note that the warp sets in where the rotation curve starts to be well approximated by a Keplerian, i.e. at the point where the exterior mass, and hence disk mass, becomes negligible. 

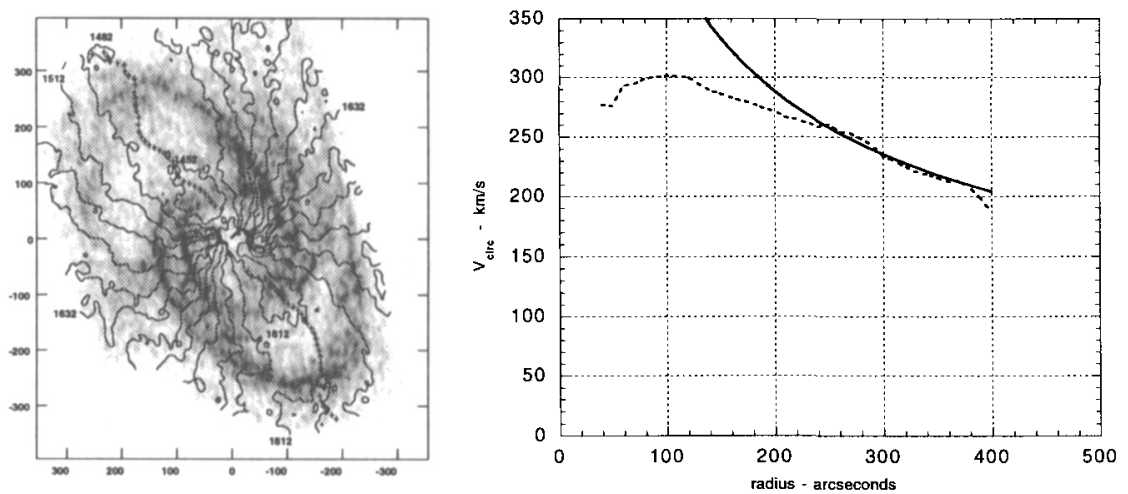

Figure 2. The velocity field superimposed on a total hydrogen map (left). The isovelocities range from 1452 to $1812 \mathrm{~km} \mathrm{~s}^{-1}$ and are separated by $30 \mathrm{~km} \mathrm{~s}^{-1}$. The crosses indicate the kinematical major axis, assuming a constant inclination of $40^{\circ}$. Right: the rotation curve (dashed) fitted to this velocity field using a tilted ring model and a Keplerian curve (solid).

\section{References}

Briggs, F. H. 1990, ApJ, 352, 15

Jörsäter, S. \& Van Moorsel, G. 1995, AJ, 110, 2037

Jörsäter, S. 1984, "The Velocity Field of NGC 1365. II. The Gas Velocity Field" Ph.D. Thesis, Stockholm Observatory

Sandqvist, Aa., Elfhag, T., \& Jörsäter, S. 1988, A\&A, 201, 223

Sandqvist, Aa., Jörsäter, S., \& Lindblad, P. O. 1994, A\&A, 295, 585 